\section{TROMBOEMBOLISMO VENOSO: PROFILAXIA EM PACIENTES CLÍNICOS - PARTE III}

Autoria: Academia Brasileira de Neurologia, Associação de Medicina Intensiva Brasileira, Federação Brasileira das Associações de Ginecologia e Obstetrícia, Sociedade Brasileira de Angiologia e de Cirurgia Vascular, Sociedade Brasileira de Cancerologia, Sociedade Brasileira de Cardiologia, Sociedade Brasileira de Clínica Médica, Sociedade Brasileira de Geriatria e Gerontologia, Sociedade Brasileira de Hematologia e Hemoterapia, Sociedade Brasileira de Pneumologia e Tisiologia, Sociedade Brasileira de Reumatologia

Participantes: Ana Thereza Rocha, Edison Ferreira de Paiva, Arnaldo Lichtenstein, Rodolfo Milani Junior, Cyrillo Cavalheiro Filho, Francisco Humberto de Abreu Maffei, Charles André, Marcia Maiumi Fukujima, Gabriel R. de Freitas, Silvia Lage, Claudio Bonduki, Carlos Carvalho, Eduardo Ramacciotti, Vera Lúcia Teixeira, Paulo Roberto Mattos da Silveira, Clarissa Mathias, José Carlos Nicolau, Renato Delascio Lopes, Salo Buksman, Verônica Hagemeyer Santos, Élbio D’Amico, Mário Terra-Filho, Roger Abramino Levy, Alexandre Wagner Silva de Souza

Descrição do método de coleta de evidência: A pesquisa envolveu os seguintes bancos de dados: a) Medline (de 1966 a agosto de 2004), b) Base de Dados Cochrane de Revisões Sistemáticas (Cochrane Library), c) LILACS (de 1985 a 2004), d) resumos de estudos publicados em encontros científicos das sociedades envolvidas, e) referências cruzadas do material levantado. A estratégia utilizada no Medline foi ("Thrombosis"[MeSH] OR "Venous Thrombosis"[MeSH] OR "Embolism and Thrombosis"[MeSH] OR "Embolism"[MeSH] OR "Pulmonary Embolism"[MeSH]) NOT "Atrial Fibrillation"[MeSH] NOT ("Surgery"[MeSH] OR "surgery"[Subheading] OR "Surgical Procedures, Operative" $[\mathrm{MeSH}]$ ) NOT ("Wounds and Injuries"[MeSH] OR "injuries"[Subheading] OR "Multiple Trauma"[MeSH])".

\section{Graus de recomendação e força de evidência:}

A: Estudos experimentais ou observacionais de melhor consistência.

B: Estudos experimentais ou observacionais de menor consistência.

C: Relatos de casos (estudos não controlados).

D: Opinião desprovida de avaliação crítica, baseada em consensos, estudos fisiológicos ou modelos animais.

\section{Introdução}

Tromboembolismo venoso (TEV) representa um espectro de doenças que inclui trombose venosa profunda, trombose associada a cateteres venosos centrais e, a complicação mais grave, tromboembolismo pulmonar (TEP). Tanto episódios assintomáticos, quanto aqueles clinicamente aparentes em pacientes hospitalizados estão associados à fatalidade. Em hospitais brasileiros e de todo o mundo, a suspeita antemortem é feita em pequena proporção dos TEP. A ênfase dada à incidência de TEV em populações cirúrgicas despertou a necessidade de profilaxia para este grupo de pacientes. No entanto, mais da metade dos pacientes que sofrem TEV sintomático não têm doenças cirúrgicas, mas sim clínicas.

Os fatores de risco para TEV em pacientes não submetidos à cirurgia e as medidas profiláticas são abordados nas diretrizes de profilaxia de TEV em pacientes clínicos, dividida em três partes. Na primeira parte, encontram-se as informações sobre: mobilidade reduzida, idade $>55$ anos, história prévia de TEV, varizes, insuficiência venosa crônica, insuficiência arterial periférica, obesidade, trombofilias hereditárias e adquiridas. Na segunda parte: gravidez, pós-parto, reposição hormonal, contraceptivos, insuficiência cardíaca congestiva classes III-IV, infarto agudo do miocárdio, acidente vascular cerebral isquêmico, acidente vascular cerebral hemorrágico, paresia e paralisia de membros inferiores. Na terceira parte: doença reumatológica ativa, doença inflamatória intestinal, doenças respiratórias graves, infecções com exceção das torácicas, síndrome nefrótica, câncer, quimioterapia, hormonioterapia, internação em unidade de terapia intensiva, uso de cateteres venosos centrais, uso de cateteres para hemodiálise, uso de Swan-Ganz. Versão integral e extensa que originou essas diretrizes encontra-se em documento elaborado pelo Grupo de Estudos de Trombose e Hemostasia.

Para identificar os estudos, uma pesquisa auxiliada por computador foi feita independentemente por cada representante das sociedades e pelos coordenadores para cada um dos assuntos do parágrafo acima. Procurou-se, também, estudos avaliando a eficácia dos métodos de profilaxia contra TEV, nesta população, incluindo heparina não fracionada (HNF), heparinas de baixo peso molecular (HBPM) e métodos físicos.

A seleção dos estudos foi baseada na leitura do título e do resumo e, quando necessário, na leitura do texto completo por pelo menos dois investigadores de cada área. A avaliação da qualidade dos estudos baseou-se em roteiros padronizados, considerando-se: desenho do estudo, randomização, recrutamento de pacientes consecutivos, seguimento apropriado, método de detecção de TEV, avaliação mascarada, precisão dos resultados e aplicabilidade dos resultados às questões clínicas sobre fatores de risco ou eficácia da profilaxia contra TEV. Como se avaliou, nesta revisão, tanto o risco quanto a eficácia de profilaxia contra TEV como desfechos principais dos estudos, foram incluídos, preferencialmente, ensaios clínicos controlados e randomizados (ECCR), mas também estudos de coorte e caso-controle.

Foram coletados: desenho do estudo, número de pacientes recrutados e analisados, características da população investigada, tipo de teste de triagem usado para detecção de TEV e incidência de TEV no grupo com o fator de risco, com e sem uso de profilaxia, se aplicável. Quando possível, foram também coletados ou calculados o risco relativo (RR) ou a razão de chances ("odds ratio" ou $\mathrm{OR}$ ), a redução do risco relativo (RRR) e o número necessário para causar danos ("number needed to harm" ou NNH), para estudos investigando risco, e o número necessário tratar ("number needed to treat" ou NNT), para estudos investigando benefícios da profilaxia, e o intervalo de confiança (IC). 


\section{Doenças reumatológicas ativas e doenças inflamatórias intestinais}

Análise de 540 pacientes encaminhados por suspeita de TVP detectou que a presença de lúpus eritematoso sistêmico (LES) foi fator de risco para o desenvolvimento de TEV (OR 4,31; IC de $95 \% 3,14-5,48)^{1}(\mathbf{C})$. A presença de anticorpos antifosfolípides como anticardiolipina e anticoagulante lúpico está associada a aumento no risco de TEV, principalmente na síndrome antifosfolípide primária e na forma secundária ao LES. Em 173 pacientes com LES, a presença de AL aumentou de maneira significativa a incidência de TEV (OR 6,4; IC de 95\% 2,7-15,4) 2 (C). Na doença de Behçet, há diversos relatos de trombose, em diferentes leitos vasculares, embora a trombose venosa profunda de membros inferiores seja a mais prevalente ${ }^{3}(\mathbf{C})$.

Estudo com 618 pacientes com doença intestinal inflamatória e 243 pacientes com doença reumatoide, comparados com igual número de controles pareados para sexo e idade, mostrou incidência maior de TEV nos primeiros (OR 3,6; IC de 95\% 1,7-7,8, $p<0,001$ ), mas não nos pacientes com doença reumatóide (OR $0,7 ; \mathrm{IC}$ de $95 \% 0,2-2,9, \mathrm{p}=\mathrm{NS})^{4}(\mathbf{B})$.

Análise de subgrupo sugere benefícios da profilaxia de TEV com heparina em doenças reumatológicas ativas ${ }^{5}(\mathbf{B})$, no entanto, é preciso comprovar em estudos especificamente desenhados e com maior número de casos. Não existem estudos especialmente dirigidos às doenças intestinais inflamatórias.

\section{Doenças respiratórias}

Doenças respiratórias como doença pulmonar obstrutiva crônica (DPOC) e pneumonia são frequentemente citadas como fatores de risco para TEV, mas estudos avaliando especificamente estas doenças são raros. O diagnóstico de TEV em pacientes com DPOC é geralmente um desafio, pois TEP pode se manifestar apenas como a piora de um estado respiratório já precário. A suspeita clínica tem pouca especificidade, já que as duas condições compartilham sintomas e sinais como dispnéia, sibilância, dor pleurítica, hemoptise, taquicardia e sinais de sobrecarga ventricular direita. Além disso, com exceção da angiografia pulmonar e, talvez, da tomografia computadorizada helicoidal com protocolo específico para TEP, a acurácia dos métodos de imagem pulmonares não invasivos tem limitações importantes nestes pacientes, devido à presença de anormalidades parenquimatosas ${ }^{6}(\mathbf{C})$.

Em coortes prospectivas com métodos diferentes de triagem de TVP, em pacientes com DPOC agudizado, a incidência de TEV vai de $9 \%$, com flebografia e/ou fibrinogênio $\operatorname{marcado}^{7}(\mathbf{C})$, para $11 \%$, com ultrassonografia com $\operatorname{Doppler}^{8}(\mathbf{C})$, e 29\%, quando são combinados cintilografia pulmonar e $\operatorname{Doppler}^{9}(\mathbf{C})$.

Existem poucos ECCRs avaliando a eficácia da profilaxia exclusivamente em pacientes com doenças pulmonares. Pacientes com DPOC exacerbado, necessitando de suporte ventilatório (a maioria com infecção pulmonar), foram randomizados para HBPM (nadroparina 3.800 UI AXa ou 5.700 UI AXa) ou placebo ${ }^{10}(\mathbf{A})$. Aqueles recebendo HBPM tiveram uma redução significativa na incidência de TEV, quando comparada ao placebo $(28,2 \%$ vs. $15,5 \%$, respectivamente, $p=0,045)$. A presença de anormalidades de testes de função pulmonar, anormalidades em gasometria arterial, ou ambos, e pelo menos uma das seguintes condições clínicas: hipertensão pulmonar secundária grave, pneumonia, doença intersticial pulmonar, câncer de pulmão e/ou metástases com expectativa de vida $>2$ meses, ou exacerbação de DPOC, caracterizam a doença respiratória grave que aumentam o risco de TEV e, portanto, é útil na avaliação global de risco dos pacientes clínicos hospitalizados.

Resumo apresentado em congresso de um estudo que incluiu, entre outros, pacientes com insuficiência respiratória sem necessidade de suporte ventilatório, demonstrou a eficácia das HBPMs, como profilaxia no grupo geral de pacientes clínicos. Um único estudo avaliou métodos mecânicos de profilaxia em pacientes com doenças respiratórias como infecção pulmonar, necessidade de suporte ventilatório, insuficiência respiratória crônica, ICC, cor pulmonale ou câncer de pulmão ${ }^{11}(\mathbf{B})$. Nesta corte prospectiva com falhas metodológicas, usou-se fibrinogênio marcado e flebografia como triagem e sugeriu-se que, tanto meia elástica de compressão gradual (MECG) ou compressão pneumática intermitente (CPI), quanto HNF (5.000 UI de 12-12 horas) levavam a uma incidência significativamente menor de TVP do que a do grupo controle.

\section{Infecções}

A análise de 1.272 pacientes ambulatoriais demonstrou que infecção é fator de risco para TEV (OR 1,95; IC de 95\% 1,31-2,92). Entretanto, o local das infecções não é relatado neste trabalho ${ }^{12}(\mathbf{B})$. Estudo com 5.451 pacientes apresentando episódios agudos de TVP, confirmado por ultrassonografia, demonstrou que $22 \%$ apresentavam alguma infecção como comorbidade: $7 \%$ tinham pneumonia, $10 \%$ outras infecções e $5 \%$ sepse, sendo que destes, 68 a 90\% estavam hospitalizados. Neste estudo, as causas mais frequentes de hospitalização foram infecções (16\%). Os autores mostraram, ainda, que infecção torácica foi mais comum em pacientes apresentando TEP em concomitância com TVP do que naqueles com TVP apenas ( $10 \%$ vs $8 \% p=0,04)$. Em outro estudo, as infecções agudas foram um dos principais fatores de risco para TEV, tanto na análise univariada (RR 1,47; IC de 95\% 1,47-2,14), quanto como fatores independentes na análise multivariada (OR 1,74; IC de 95\% 1,12-2,75) ${ }^{13}(\mathbf{B})$. Estes dados sugerem que infecções são fatores de risco adicional para TEV, por representarem comorbidades frequentes nestes pacientes, principalmente na população hospitalizada. As infecções torácicas parecem ser fatores de risco para TEV mais importante que as não torácicas. Quanto à eficácia da profilaxia de $T E V$, em pacientes com infecções, a análise da evidência foi feita de maneira indireta, por se tratar apenas de subgrupos de estudos com pacientes clínicos em geral, e não como objetivo primário dos estudos. Em um estudo multicêntrico, prospectivo e controlado, mas com algumas falhas metodológicas, foram randomizados 11.693 pacientes internados em enfermarias de infectologia para HNF $5.000 \mathrm{UI}$ de 12-12 horas ou nenhum tratamento. O objetivo primário deste estudo foi também o índice de mortalidade, que não diferiu entre os grupos tratados e controle (5,3 vs. $5,6 \%, p=0.39)$. Os métodos de detecção de TEV não fatal não foram descritos, porém, as complicações tromboembólicas não fatais foram significativamente menos frequentes em 
pacientes recebendo profilaxia (70/5.776 vs. $116 / 5.917, p=$ $0,0012)^{14}(\mathbf{A})$.

\section{Síndrome nefrótica}

A incidência global de TEV na síndrome nefrótica é de $43 \%{ }^{15}$ (D), sendo que tanto TEP quanto TVP afetam cerca de $11 \%$ dos pacientes. A frequencia estimada de trombose de veia renal, na síndrome nefrótica membranosa, em adultos, varia de $5 \%$ a $60 \%{ }^{16}(\mathbf{D})$. Destes episódios de trombose de veia renal, apenas $10 \%$ são sintomáticos. Tanto as tromboses sintomáticas quanto as assintomáticas podem ser confirmadas acuradamente com tomografia computadorizada ${ }^{17}(\mathbf{C})$. Revisão da literatura com 13 trabalhos prospectivos, totalizando 682 pacientes com síndrome nefrótica, mostrou incidência de trombose de veia renal de $21,4 \%$ (IC de 95\% 18-25\%). Em três trabalhos prospectivos, com 148 pacientes com síndrome nefrótica, encontrou-se incidência de TEP de 14\% (IC de 95\% 9-21\%)18(B). No grupo de cinco estudos com síndrome nefrótica em geral, entre 321 pacientes, 64 casos apresentaram trombose (20\%). Entre os nove estudos de pacientes com síndrome nefrótica membranosa, houve 70 eventos de trombose de veia renal, entre 216 pacientes $(32 \%)^{19}(\mathbf{D})$.

Um estudo prospectivo que avaliou a enoxaparina (40mg diários) na prevenção de TEV, em 65 pacientes nefróticos ambulatoriais, mostrou na avaliação inicial de 10 pacientes, após seguimento por 3 a 12 meses que não houve nenhum evento trombótico revelado por ultrassonografia com Doppler das veias renais e membros inferiores, e cintilografia pulmonar, realizados a cada três meses ${ }^{18}(\mathbf{B})$.

\section{Neoplasias, quimioterapia e hormonioterapia}

Estudo de 366 episódios de TEV entre duas cortes com 21.680 pacientes, seguidos por uma média de 7,6 anos, mostrou que, entre os 191 pacientes com episódios de TEV considerados secundários, 48\% tinham câncer. Além disso, câncer foi o único fator independentemente associado a mortalidade em 28 dias (RR 5,2, IC de 95\% 1,4-19,9) e trombose recorrente (RR 9,2; IC de 95\% 2,0-41,7)20(B). O acompanhamento de 61.998 pacientes com TEV por 18 anos encontrou aumento de quatro vezes no risco de câncer, após um ano do diagnóstico de TEV. Nesta corte, os cânceres mais frequentes foram fígado, pâncreas, ovário, cérebro, linfoma de Hodgkin e policitemia vera. Além disto, o risco de desenvolvimento de câncer foi maior em pacientes mais jovens do que nos mais idosos. A incidência de câncer persistia alta no decorrer de até $10 \operatorname{anos}^{21}(\mathbf{B})$. 0 seguimento de 250 pacientes com TVP, durante dois anos, mostrou que câncer sintomático acometeu significativamente mais pacientes com TVP idiopática $(7,6 \%)$, que pacientes com TVP secundária (1,9\%), OR 2,3 (IC de $95 \% 1,0-5,2 ; p=0,04)$. Além disso, $24 \%$ daqueles com TVP idiopática tiveram recorrência de TEV, e destes, 17,1\% desenvolveram câncer. Chama-se a atenção para a uma maior chance de desenvolvimento de câncer naqueles com TEV idiopático e recorrente, quando comparados a pacientes com TEV secundário (OR 9,8, IC de 95\% 1,8-52,2, $p=0,008$ ), e também a pacientes com TEV idiopático, mas não recorrente (OR 4,3, IC de 95\% 1,2-15,3, $p=0,02)^{22}$ (B). Portanto, em pacientes diagnosticados com TEV idiopático, particularmente naqueles com episódios recorrentes, a presença de câncer deve ser considerada entre os diagnósticos diferenciais.

Em pacientes com câncer de mama, a incidência de TEV durante o período de quimioterapia é bastante superior à encontrada no período sem tratamento, variando de $6,8 \%$ a $17,6 \%$, dependendo do estágio da doença e das medicações do esquema de tratamento. O uso de tamoxifeno mais quimioterapia aumentou o risco de TEV, quando comparado à utilização da quimioterapia isolada, tanto no grupo de mulheres pré-menopausadas $(2,8$ vs $0,8 p=0,03)$ quanto nas mulheres menopausadas $(8.0 \%$ vs $2.3 \%, p=0.03)^{23}(\mathbf{A})$. Em estudo de pacientes com mieloma múltiplo, a adição de talidomida ao esquema poliquimioterápico se associou a aumento na incidência de TVP (28\% vs. $4 \%$, $\mathrm{p}=0,002)^{24}(\mathbf{A})$.

Quanto à profilaxia de TEV, existem poucos estudos avaliando diferentes anticoagulantes em pacientes com câncer, principalmente durante o período de tratamento. 0 efeito da anticoagulação com warfarina em homens com neoplasia de pequenas células de pulmão se associou a aumento da sobrevida $(p=$ 0,018 ) e a maior tempo até que a progressão da doença se tornasse evidente $(p=0,016)$. Este fato não ocorreu com outras neoplasias estudadas ${ }^{25}(\mathbf{B})$. Estudo de corte com 256 pacientes com mieloma múltiplo sugeriu que o uso de HBPM (enoxaparina $40 \mathrm{mg} / \mathrm{dia}$ ) diminui a incidência de TVP relacionada ao uso de talidomida mais poliquimioterapia ${ }^{26}(\mathbf{A})$. Estudo randomizado com pacientes portadores de neoplasias avançadas para receber dalteparina (5.000 UI/dia) ou placebo por um ano mostrou que a sobrevida, as incidências de TEV sintomática e sangramento não foram afetadas pelo tratamento ${ }^{27}$ (B). Em pacientes com câncer e episódio agudo de TVP, TEP ou ambos, a HBPM dalteparina utilizada diariamente por seis meses foi superior à anticoagulação com warfarina (RNI 2,5) na prevenção da recorrência de TEV (8\% vs. $16 \%, p=0,002)^{28}(\mathbf{A})$.

\section{UTI}

A admissão em UTI é fator de risco para $\operatorname{TEV}^{29}(\mathbf{C})$, especialmente em pacientes que não recebem profilaxia. Embora pacientes em UTI tenham vários outros fatores de risco adicionais para TEV, não incluindo imobilização, a utilização de profilaxia, nestes pacientes, ainda é bastante irregular.

Quanto à eficácia da quimioprofilaxia, existem duas revisões sistemáticas sobre o tema, mas que avaliaram apenas quatro ECCRs e destes, três foram publicados somente como "abstracts" ${ }^{30}(\mathbf{A})$. Nos dois primeiros ECCRs, HNF (5.000 UI de 12-12 h) foi comparada com placebo e se mostrou eficaz, com uma RRR de TEV de $55 \%$, em um e de $65 \%$, no outro estudo ${ }^{31}(\mathbf{A})$. De acordo com estes estudos, seria necessário tratar cinco ou seis pacientes, com HNF, para reduzir o risco de TEV em um deles. Estudo multicêntrico divulgado em congresso, triando 11.000 pacientes, recrutou 325 pacientes de UTIs clínicas, que foram então randomizados para receber HNF (5.000 UI de 12-12 h) ou enoxaparina (30 mg de 12-12 h). A avaliação ultrassonográfica sequencial de TVP demonstrou que os dois regimes foram 
igualmente efetivos e seguros. A literatura é ainda mais escassa quanto a estudos sobre métodos mecânicos de profilaxia, para pacientes em UTIs. Existe apenas um ECCR também publicado na forma resumida para congresso, avaliando a eficácia de CPI, em pacientes de UTI clínica com contraindicações para heparina. Neste, os autores mostraram que, de acordo com os achados ultrasonográficos com Doppler no primeiro e último dias de internação, CPI foi tão ineficaz quanto a falta de profilaxia, na prevenção de TVP (37\% vs. 31\%, respectivamente).

Estudo de corte com 192 pacientes clínicos de alto risco para TEV, recebendo regimes variados de profilaxia, triando-os com fibrinogênio marcado, ultrassonografia e pletismografia, mostrou que a incidência de TVP foi significativamente menor naqueles recebendo HNF 5.000 UI de $12-12$ h (3\% vs. $26 \%, p<0,002$ ) ou MECG, usadas 24 horas por dia (0\% vs. $26 \%, p<0,0003$ ), do que no grupo controle ${ }^{11}(\mathbf{B})$. Outro estudo de coorte, com teste de triagem para TVP, também sugeriu a utilidade da HNF ou de $\mathrm{CPI}$, quando comparadas com placebo ( $12 \%$ vs. $25 \%$ ), mas a diferença não foi significativa ${ }^{29}(\mathbf{C})$. Em outra comunicação de congresso, a incidência de TEV sintomático foi semelhante entre 15.499 pacientes que receberam CPI $(1,3 \%)$ ou HNF ou HBPM (1\%). De um modo geral, HNF ou HBPMs são eficazes, quando comparadas à placebo, e estão indicadas para pacientes de UTIs clínicas. Entretanto, mesmo com profilaxia, a incidência de TEV ainda é $\operatorname{alta}^{32}(\mathbf{B})$ e parece similar entre aqueles recebendo HNF ou HBPM.

\section{Cateteres venosos}

Diversas variáveis estão associadas ao aumento no risco de trombose de cateteres venosos centrais (Trombose-CVC) e TVP, em pacientes portadores de cateteres. Os estudos avaliando prevenção de trombose nestes pacientes são bastante heterogêneos. Apresentaremos a evidência sobre cateteres venosos centrais de acordo com o propósito do cateter: para quimioterapia em pacientes com câncer, para nutrição parenteral (NP) e para uso geral em pacientes de UTI. Em seguida, discutiremos os cateteres de artéria pulmonar e os cateteres para hemodiálise como fatores de risco para TEV.

A incidência de Trombose-CVC, em pacientes sem profilaxia, é de $4 \%$ a $62 \%$ vs. $5 \%$ a $18 \%$ naqueles recebendo profilaxia. Portanto, cateteres venosos centrais constituem um fator de risco adicional para TEV, na população em geral ${ }^{33}(\mathbf{B})$, e em pacientes oncológicos, em particular ${ }^{34}(\mathbf{B})$.

Quanto ao uso de profilaxia para Trombose-CVC em pacientes com câncer e cateteres, há controvérsia. No estudo pioneiro, no qual 121 pacientes com câncer foram randomizados para receber ou não dose baixa de warfarina ( $1 \mathrm{mg} / \mathrm{dia}$ ), a profilaxia mostrou-se eficaz contra Trombose-CVC (9,5\% vs. 37,5\%, p<0,001) ${ }^{35}(\mathbf{A})$. Neste estudo, o objetivo final foi trombose diagnosticada por flebografia, realizada caso houvesse sintomas de trombose, ou na conclusão do estudo (90 dias), nos demais pacientes. Por outro lado, os dois estudos que vêm desafiar estes achados apresentam importantes falhas metodológicas. Em um $\operatorname{deles}^{36}(\mathbf{B})$, 88 pacientes com 102 cateteres foram randomizados para warfarina ( $1 \mathrm{mg} / \mathrm{dia}$ ) ou controle e a incidência de trombose não foi diferente entre os grupos (17,8\% e $11,6 \%$, respectivamente,
$p=N S$ ). Porém, neste estudo, pacientes com CVC ou TromboseCVC prévios e, portanto, com riscos diferentes, não foram excluídos. Assim, depois de constatar que os 11 pacientes necessitando de dois ou mais cateteres apresentavam incidência de trombose muito mais alta que os demais $(35,7 \%)$, os cateteres secundários foram excluídos da análise post-hoc. Além disso, os pacientes que deveriam receber warfarina por 90 dias só o fizeram, em média, por 41 dias. No outro estudo, publicado apenas como "abstract", um número maior de pacientes com câncer $(n=255)$ foi também randomizado para warfarina ( $1 \mathrm{mg} / \mathrm{dia}$ ) ou placebo. Não houve diferença significativa na incidência de TromboseCVC (4,6\% vs. $4,0 \%, p=N S)$ ou sangramento entre os grupos. Entretanto, neste estudo, 75\% dos pacientes não concluíram o tratamento (a maioria por causa de trombocitopenia) e o dobro de pacientes no grupo placebo teve os cateteres removidos por mau funcionamento, um desfecho importante que não foi considerado. Além disto, em ambos os estudos, a detecção de trombose foi feita apenas quando sintomas de oclusão ou mau funcionamento estavam presentes. Por outro lado, a concomitância de metástases hepáticas e o uso do quimioterápico fluoracil e derivados, durante a profilaxia com warfarina em baixa dose, têm sido associados a elevações na RNI e a uma incidência elevada de sangramento $(8 \%)^{36}(\mathbf{B})$.

Quanto à HBPM para profilaxia de Trombose-CVC, existe um ECCR pequeno, comparando dalteparina (2.500 UI/dia) com placebo, em pacientes com câncer e cateteres ${ }^{37}(\mathbf{B})$. Mostra-se que há uma redução estatisticamente significante nos riscos absoluto (55\%) e relativo $(90 \%)$ de Trombose-CVC detectada com flebografia.

Duas metanálises compilam estudos de profilaxia em pacientes com cateteres. A primeira combina diversos ECCRs que avaliam HNF e HBPM subcutâneas, em pacientes com câncer, e também regimes de heparina endovenosa para redução de Trombose-CVC, em pacientes recebendo $\mathrm{NP}^{38}(\mathbf{A})$. Apesar da avaliação incluir tanto estudos de pacientes adultos quanto pediátricos, estimam uma redução do risco relativo de Trombose-CVC de 57\% (RR 0,44; IC de 95\% 0,22-0,87), a partir de seis estudos em adultos recebendo HNF ( $1 \mathrm{U} / \mathrm{mL}$ ou $3 \mathrm{U} / \mathrm{mL}$ adicionados à NP, 5.000 Ul de 6-6 h ou 12-12 h) ou HBPM (dalteparina $2.500 \mathrm{UI} / \mathrm{dia}$ ). A segunda metanálise, com estudos separados de acordo com o propósito do cateter e a população de pacientes $^{39}(\mathbf{A})$, mostrou que, baseando-se em cinco ECCRs e uma corte prospectiva, HNF ( $1 \mathrm{U} / \mathrm{mL}$ ou $3 \mathrm{U} / \mathrm{mL}$ adicionados à NP, ou $5.000 \mathrm{UI}$ de 6-6 h) não é eficaz na redução de Trombose-CVC, em pacientes recebendo NP (RR 0,77; IC de 95\% 0,11-5,48). Para pacientes de UTI com cateteres, os autores concluem que existe evidência insuficiente sobre a utilidade de profilaxia para redução risco de TromboseCVC. Finalmente, para pacientes com câncer e cateteres, estes autores concluem que, tanto dose baixa de warfarina, quanto HBPM, são eficazes na redução risco de Trombose-CVC (RR 0,25 e 0,10 , respectivamente).

\section{Cateter de artéria pulmonar (Swan-Ganz)}

A formação de trombose em cateteres de artéria pulmonar é dependente do tempo de permanência e vai de 66\% a quase $100 \%$ em cateteres não impregnados com heparina. Entretanto, a 
frequencia de complicações trombóticas varia consideravelmente, dependendo do método de detecção do $\operatorname{trombo}^{40}(\mathbf{C})$. Assim, como outros CVC, cateteres de artéria pulmonar aumentam o risco de trombose da veia cateterizada, em comparação às veias contralaterais ${ }^{41}(\mathbf{C})(R R 4,5)$, e constituem, portanto, um fator de risco adicional para TEV. Uma metanálise ${ }^{42}(\mathbf{A})$ mostra que os cateteres impregnados com heparina reduzem o risco de Trombose-CVC em 92\%, mas apenas durante as primeiras 24 horas (RR 0,08; IC de 95\%, 0,02-0,37). Não existem estudos investigando, especificamente, a quimioprofilaxia com heparinas, em pacientes com cateteres de artéria pulmonar e, desta forma, nenhuma recomendação específica pode ser feita.

\section{Cateter para hemodiálise}

Apesar da insuficiência renal estar associada a um risco aumentado de sangramento, eventos trombóticos também são bastante comuns em pacientes com falência renal. Pacientes recebendo hemodiálise crônica têm incidência elevada de trombofilias, frequentemente utilizam eritropoetina recombinante que pode ter efeito pró-trombótico e apresentam fatores de risco para TEV que são menos reconhecidos, como hiperhomocisteinemia, disfunção endotelial e marcadores inflamatórios sistêmicos. Na análise de um banco de dados americano com 76.718 pacientes com falência renal, em diálise crônica, em 1996, a incidência de TEP foi de 149,9/100.000, comparada com 24,6/100.000 na população geral dos Estados Unidos ${ }^{43}(\mathbf{B})$. Além disso, trombose do acesso venoso é outra condição bem estabelecida nestes pacientes, afetando tanto as fístulas e enxertos, quanto os cateteres de duplo lúmen para hemodiálise. No entanto, o mau funcionamento não é incomum e a incidência de trombose-CVC, nestes pacientes, chega a $46 \%^{44}(\mathbf{C})$, sendo rara, entretanto, a necessidade de sua remoção. Existe, também, evidência de que a canulação de veias subclávias causa mais trombose e/ou estenose do que a canulação de veias jugulares internas, particularmente no lado esquerdo ${ }^{45}(\mathbf{C})$.

Alguns estudos pequenos com aspirina (AAS), sulfinpirazona ${ }^{46}(\mathbf{B})$, dipiridamol ${ }^{47}(\mathbf{A})$ e ticlopidina ${ }^{48}(\mathbf{B})$ sugerem a eficácia destes agentes na prevenção de trombose de enxertos e fístulas para hemodiálise. Depois de recrutar 200 pacientes em diálise crônica, um estudo para profilaxia de trombose de enxertos vasculares foi interrompido precocemente, devido a um maior risco de sangramento no grupo aspirina e clopidogrel comparado ao placebo ( $24 \%$ vs. $42 \%, p<0,006$ ) e, portanto, não houve poder suficiente para determinar a eficácia do tratamento ${ }^{49}(\mathbf{A})$. Nenhum estudo controlado avaliou, especificamente, a quimioprofilaxia contra TEV ou trombose-CVC, em pacientes com cateteres para hemodiálise. Nestes pacientes, a obstrução do cateter pode ser reduzida pelo posicionamento correto da sua ponta no átrio direito ${ }^{50}(\mathbf{C})$. Além disso, agentes trombolíticos como uroquinase e estreptoquinase têm conseguido restabelecer a patência do cateter em mais de $90 \%$ dos episódios de trombose, evitando-se assim a necessidade de remoção do mesmo ${ }^{47}(\mathbf{A})$. Existe evidência insuficiente para recomendar o uso de quimioprofilaxia contra TEV em pacientes dialisados através de cateteres temporários.
Referências

1. Cogo A, Bernardi E, Prandoni P, Girolami B, Noventa F, Simioni P, et al. Acquired risk factors for deep-vein thrombosis in symptomatic outpatients. Arch Intern Med 1994;154: 164-8.

2. Fijnheer R, Horbach DA, Donders RC, Vile H, von Oort E, Nieuwenhuis HK, et al. Factor $V$ Leiden, antiphospholipid antibodies and thrombosis in systemic lupus erythematosus. Thromb Haemost 1996; 76: 514-7.

3. Koc Y, Gullu I, Akpek G, Akpolat T, Kanser E, Kiraz S, et al. Vascular involvement in Behcet's disease. J Rheumatol 1992; 19: 402-10.

4. Miehsler W, Reinisch W, Valic E, Osterode W, Tillinger W, Feichtenschlager $\mathrm{T}$, et al. Is inflammatory bowel disease an independent and disease specific risk factor for thromboembolism? Gut 2004; 53: 542-8.

5. Alikhan R, Cohen AT, Combe S, Samama MM, Desjardins L, Eldor A, et al. Prevention of venous thromboembolism in medical patients with enoxaparin: a subgroup analysis of the MEDENOX study. Blood Coagul Fibrinolysis 2003; 14: 341-6.

6. Lesser BA, Leeper KV Jr, Stein PD, Saltzman HA, Chen J, Thompson BT, et al. The diagnosis of acute pulmonary embolism in patients with chronic obstructive pulmonary disease. Chest 1992; 102: 17-22.

7. Prescott SM, Richards KL, Tikoff G, Armstrong JD Jr, Shigeoka JW. Venous thromboembolism in decompensated chronic obstructive pulmonary disease. A prospective study. Am Rev Respir Dis 1981; 123: 32-6.

8. Erelel M, Cuhadaroglu C, Ece T, Arseven O. The frequency of deep venous thrombosis and pulmonary embolus in acute exacerbation of chronic obstructive pulmonary disease. Respir Med 2002; 96: 515-8.

9. Mispelaere D, Glerant JC, Audebert M, Remond A, Sevestre-Pietri MA, Jounicaux V. Pulmonary embolism and sibilant types of chronic obstructive pulmonary disease decompensations. Rev Mal Respir 2002; 19: 415-23.

10. Fraisse F, Holzapfel L, Couland JM, Simonneau G, Bedock B, Feissel M, et al. Nadroparin in the prevention of deep vein thrombosis in acute decompensated COPD. The Association of Non-University Affiliated Intensive Care Specialist Physicians of France. Am J Respir Crit Care Med 2000; 161: 1109-14.

11. Ibarra-Perez C, Lau-Cortes E, Colmenero- Zubiate S, Arevila-Ceballos $\mathrm{N}$, Fong JH, Sanchez-Martinez R, et al. Prevalence and prevention of deep venous thrombosis of the lower extremities in high-risk pulmonary patients. Angiology 1988; 39: 505-13.

12. Samama MM. An epidemiologic study of risk factors for deep vein thrombosis in medical outpatients: the Sirius study. Arch Intern Med 2000; 160: 3415-20

13. Alikhan R, Cohen AT, Combe S, Samana MM, Desjardins L, Eldor A, et al. Risk factors for venous thromboembolism in hospitalized patients with acute medical illness: analysis of the MEDENOX Study. Arch Intern Med 2004; 164: 963-8.

14. Gardlund B. Randomised, controlled trial of low-dose heparin for prevention of fatal pulmonary embolism in patients with infectious diseases. The Heparin Prophylaxis Study Group. Lancet 1996; 347: 1357-61.

15. Bellomo R, Atkins RC. Membranous nephropathy and thromboembolism: is prophylactic anticoagulation warranted? Nephron 1993; 63: 249-54.

16. Nickolas TL, Radhakrishnan J, Appel GB. Hyperlipidemia and thrombotic complications in patients with membranous nephropathy. Semin Nephrol 2003; 23: 406-11.

17. Gatewood OM, Fishman EK, Burrow CR, Walker WG, Goldman SM, Siegelman SS. Renal vein thrombosis in patients with nephrotic syndrome: CT diagnosis. Radiology 1986; 159: 117-22.

18. Rostoker G, Durand-Zaleski I, Petit-Phar M, Ben Maadi A, Jazaerli N, Radier $\mathrm{C}$, et al. Prevention of thrombotic complications of the nephrotic syndrome by the lowmolecular-weight heparin enoxaparin. Nephron 1995; 69: 20-8.

19. Llach F. Hypercoagulability, renal vein thrombosis, and other thrombotic complications of nephrotic syndrome. Kidney Int 1985; 28: 429-39.

20. Cushman M, Tsai AW, White RH, Hechbert SR, Rosamond WD, Enright P. Deep vein thrombosis and pulmonary embolism in two cohorts: the longitudinal investigation of thromboembolism etiology. Am J Med 2004; 117: 19-25.

21. Baron JA, Gridley G, Weiderpass E, Nyren O, Linet M. Venous thromboembolism and cancer. Lancet 1998; 351: 1077-80. 
22. Prandoni P, Lensing AW, Buller HR, Cogo A, Prins MH, Catelan AM, et al. Deepvein thrombosis and the incidence of subsequent symptomatic cancer. N Engl J Med 1992;327:1128-33.

23. Saphner T, Tormey DC, Gray R. Venous and arterial thrombosis in patients who received adjuvant therapy for breast cancer. J Clin Oncol 1991; 9: 286-94. 24. Zangari M, Anaissie E, Barlogie B, Badros A, Desikan R, Gopal AV, et al. Increased risk of deep-vein thrombosis in patients with multiple myeloma receiving thalidomide and chemotherapy. Blood 2001; 98: 1614-5.

25. Zacharski LR, Henderson WG, Rickles FR, Forman WB, Cornell CJ Jr, Forcier RJ, et al. Effect of warfarin anticoagulation on survival in carcinoma of the lung, colon, head and neck, and prostate. Final report of VA Cooperative Study \#75. Câncer 1984; 53: 2046-52.

26. Zangari M, Barlogie B, Anaissie E, Saghafifar F, Eddlemen P, Jacobson $\mathrm{J}$, et al. Deep vein thrombosis in patients with multiple myeloma treated with thalidomide and chemotherapy: effects of prophylactic and therapeutic anticoagulation. Br J Haematol 2004; 126: 715-21.

27. Kakkar AK, Levine MN, Kadziola Z, Lemoine NR, Low V, Patel HK, et al. Low molecular weight heparin, therapy with dalteparin, and survival in advanced cancer: the fragmin advanced malignancy outcome study (FAMOUS). J Clin Oncol 2004; 22:1944-8.

28. Lee AY, Levine MN, Baker RI, Bowden C, Kakkar AK, Prins M, et al. Lowmolecularweight heparin versus a coumarin for the prevention of recurrent venous thromboembolism in patients with cancer. N Engl J Med 2003; 349: 146-53.

29. Marik PE, Andrews L, Maini B. The incidence of deep venous thrombosis in ICU patients. Chest 1997; 111: 661-4.

30. Geerts W, Cook D, Selby R, Etchells E. Venous thromboembolism and its prevention in critical care. J Crit Care 2002; 17: 95-104.

31. Attia J, Ray JG, Cook DJ, Douketis J, Ginsberg JG, Geerts WH. Deep vein thrombosis and its prevention in critically ill adults. Arch Intern Med 2001; 161: 1268-79.

32. Ibrahim EH, Iregui M, Prentice D, Sherman G, Kollef MH, Shannon W. Deep vein thrombosis during prolonged mechanical ventilation despite prophylaxis. Crit Care Med 2002; 30: 771-4.

33. Heit JA, Silverstein MD, Mohr DN, Petterson TM, O'Fallon WM, Melton LJ 3rd. Risk factors for deep vein thrombosis and pulmonary embolism: a populationbased case-control study. Arch Intern Med 2000; 160: 809-15.

34. De Cicco M, Matovic M, Balestreri L, Panarello G, Fantin D, Morassut S, et al. Central venous thrombosis: an early and frequent complication in cancer patients bearing long-term silastic catheter. A prospective study. Thromb Res 1997; 86: 101-13.

35. Bern MM, Lokich JJ, Wallach SR, Bothe A Jr, Benotti DN, Arkin CF, et al. Very low doses of warfarin can prevent thrombosis in central venous catheters. A randomized prospective trial. Ann Intern Med 1990; 112: 423-8.

36. Masci G, Magagnoli M, Zucali PA, Castagna L, Cornaghi C, Sarina B, et al. Minidose warfarin prophylaxis for catheterassociated thrombosis in cancer patients: can it be safely associated with fluorouracilbased chemotherapy? J Clin Oncol 2003; 21: 736-9.
37. Monreal M, Alastrue A, Rull M, Mira X, Muxart J, Rossell R, et al. Upper extremity deep venous thrombosis in cancer patients with venous access devices - prophylaxis with a low molecular weight heparin (Fragmin). Thromb Haemost 1996; 75: 251-3.

38. Randolph AG, Cook DJ, Gonzales CA, Andrew M. Benefit of heparin in central venous and pulmonary artery catheters: a meta-analysis of randomized controlled trials. Chest 1998; 113: 165-71.

39. Klerk CP, Smorenburg SM, Buller HR. Thrombosis prophylaxis in patient populations with a central venous catheter: a systematic review. Arch Intern Med 2003; 163: 1913-21.

40. Elliott CG, Zimmerman GA, Clemmer TP. Complications of pulmonary artery catheterization in the care of critically ill patients. A prospective study. Chest 1979; 76: 647-52

41. Meredith JW, Young JS, O'Neil EA, Snow DC, Hansen KJ. Femoral catheters and deep venous thrombosis: a prospective evaluation with venous duplex sonography. J Trauma 1993; 35: 187-90.

42. Randolph AG, Cook DJ, Gonzales CA, Andrew M. Benefit of heparin in peripheral venous and arterial catheters: systematic review and meta-analysis of randomized controlled trials. BMJ 1998; 316: 969-75.

43. Tveit DP, Hypolite IO, Hshieh P, Cruess D, Agodoa LY, Welch PG, et al. Chronic dialysis patients have high risk for pulmonary embolism. Am J Kidney Dis 2002; 39: 1011-7.

44. Beenen L, van Leusen R, Deenik B, Bosch FH. The incidence of subclavian vein stenosis using silicone catheters for hemodialysis. Artif Organs 1994; 18: 289-92.

45. Clark DD, Albina JE, Chazan JA. Subclavian vein stenosis and thrombosis: a potential serious complication in chronic hemodialysis patients. Am J Kidney Dis 1990; $15: 265-8$.

46. Domoto DT, Bauman JE, Joist JH. Combined aspirin and sulfinpyrazone in the prevention of recurrent hemodialysis vascular access thrombosis. Thromb Res 1991; 62: 737-43

47. Sreedhara R, Himmelfarb J, Lazarus JM, Hakim RM. Anti-platelet therapy in graft thrombosis: results of a prospective, randomized, double-blind study. Kidney Int 1994; 45: 1477-83.

48. Kobayashi K, Maeda K, Koshikawa S, Kawaguchi Y, Shimizu N, Naito C et al. Antithrombotic therapy with ticlopidine in chronic renal failure patients on maintenance hemodialysis - a multicenter collaborative double blind study. Thromb Res 1980; 20: 255-61.

49. Kaufman JS, O'Connor TZ, Zhang JH, Cronin RE, Fiore LD, Ganz MB, et al. Randomized controlled trial of clopidogrel plus aspirin to prevent hemodialysis access graft thrombosis. J Am Soc Nephrol 2003; 14: 2313-21.

50. Uldall R, Besley ME, Thomas A, Salter S, Nuezca LA, Vas M. Maintaining the patency of double-lumen silastic jugular catheters for haemodialysis. Int J Artif Organs 1993; 16: 37-40.

O texto completo da Diretriz: Tromboembolismo Venoso: Profilaxia em Pacientes Clínicos - Parte III está disponível nos sites: www.projetodiretrizes.org.br e www.amb.org.br. 